\title{
SPECTRA OF COMPACT COMPOSITION OPERATORS
}

\author{
JAMES G. CAUGHRAN AND HOWARD J. SCHWARTZ
}

ABSTRACT. Let $\phi$ be holomorphic and map the open unit disk into itself, and let $C_{\phi}: f \rightarrow f \circ \phi$ be the composition operator on $H^{2}$ generated by $\phi$. If $C_{\phi}$ is a compact operator then (1) $\phi\left(z_{0}\right)=z_{0}$ for some $z_{0} \in D$; (2) $\sigma\left(C_{\phi}\right)=\left\{\phi^{\prime}\left(z_{0}\right)^{n}: n=0,1,2, \cdots\right\} \cup\{0\}$.

Let $\phi$ be holomorphic and nonconstant in the open unit disk $\mathbf{D}$ and suppose $|\phi(z)|<1$ for $z \in \mathbf{D}$. For $f$ holomorphic in $\mathbf{D}$, define the composition operator $C_{\phi}$ by $\left(C_{\phi} f\right)(z)=(f \circ \phi)(z)=f(\phi(z))$. Then $C_{\phi}$ is a bounded linear operator on the Hilbert space $H^{2}$ [6].

In this paper, we describe the spectrum of $C_{\phi}$ in the case in which $C_{\phi}$ is compact. (For a discussion of geometric conditions which guarantee compactness, see [7].) To describe the spectrum we prove a theorem which may be of independent interest: that if $C_{\phi}$ is compact, $\phi$ has a fixed point in $\mathbf{D}$.

We are grateful to several mathematicians for conversations about this material, including James Deddens, Alan Lambert, Peter Rosenthal, and Joel Shapiro. The first author would like to express his appreciation to the National Research Council of Canada for partial support while this work was in progress.

The reproducing kernel for $H^{2}, k_{\zeta}(z)=(1-\bar{\zeta} z)^{-1}, \zeta \in \mathbf{D}$, is characterized by the property that for $f \in H^{2},\left(f, k_{\zeta}\right)=f(\zeta)$. The function $k_{\zeta}$ is itself an $H^{2}$ function, of norm $\left(1-|\zeta|^{2}\right)^{-1 / 2}$. In terms of these functions, we can characterize the composition operators among operators on $H^{2}$.

Theorem 1. Let $A$ be an operator on $H^{2}$. Then $A$ is a composition operator if and only if the image of every kernel function under $A^{*}$ is a kermel function. If $\zeta \in D, C_{\phi}^{*} k_{\zeta}=k_{\phi(\zeta)}$.

Proof. If $A=C_{\phi}$ is a composition operator, then

Presented to the Society, April 1, 1972 under the title Spectra of composition operators on $\mathrm{H}^{2}$; received by the editors December 26, 1973.

AMS (MOS) subject classifications (1970)。 Primary 30A78, 47A10; Secondary $30 \mathrm{~A} 31,47 \mathrm{~B} 05$. 


$$
\left(f, A^{*} k_{\zeta}\right)=\left(C_{\phi} f, k_{\zeta}\right)=f(\phi(\zeta))=\left(f, k_{\phi(\zeta)}\right),
$$

so $A^{*} k_{\zeta}=k_{\phi(\zeta)}$ for every $\zeta \in D$.

Conversely, if $A^{*}$ takes each kernel function $k_{\zeta}$ into a kernel function $k_{\phi(\zeta)}$, then if $f \in H^{2}$,

$$
(A f)(\zeta)=\left(A f, k_{\zeta}\right)=\left(f, A^{*} k_{\zeta}\right)=\left(f, k_{\phi(z)}\right)=f(\phi(\zeta)) .
$$

Letting $f$ be the identity function $f(z)=z$, we see $\phi$ is holomorphic and $A=C_{\phi}$.

Theorem 2. If $C_{\phi}$ is a compact composition operator, then $\phi(z)$ has a fixed point in $\mathbf{D}$.

Proof. Since $C_{\phi}$ is compact, $\phi$ is not a rotation about a fixed point [7]. By the Wolff-Denjoy theorem [2], [8], there is a point $a \in \overline{\mathbf{D}}$ so that for every $z \in \mathbf{D}$, the iterates $\phi_{n}(z)$ converge to $a$, where $\phi_{0}(z)=z, \phi_{n+1}(z)=$ $\phi\left(\phi_{n}(z)\right)$. If $|\alpha|<1$, then $\phi(\alpha)=\alpha$.

Suppose, by way of contradiction, that $|\alpha|=1$. Since $\lim _{n \rightarrow \infty} \phi_{n}(0)=\alpha$, for infinitely many $n,\left|\phi_{n+1}(0)\right|>\left|\phi_{n}(0)\right|$. Choose points $\zeta_{n}=\phi_{n}(0)$ from among these values of $n$. Let $k_{\zeta_{n}}$ be the kernel function for $\zeta_{n}$. Then $C_{\phi}^{*} k_{\zeta_{n}}=k_{\zeta_{n+1}}$. Because $C_{\phi}^{*}$ is compact, the images of $k_{\zeta_{n}} /\left\|k_{\zeta_{n}}\right\|$ under $C_{\phi}^{*}$ have a subsequence $\left\{g_{j}\right\}$ which converges in norm. Since

$$
\left\|g_{j}\right\|=\left\|k_{\zeta_{n+1}}\right\| /\left\|k_{\zeta_{n}}\right\|=\left(1-\left|\phi_{n}(0)\right|^{2}\right)^{1 / 2} /\left(1-\left|\phi_{n+1}(0)\right|^{2}\right)^{1 / 2}>1 \text {, }
$$

the norm of the limit function $g$ is at least 1 .

On the other hand,

$$
\begin{aligned}
\left(g, g_{j}\right) & =\left(1-\left|\zeta_{n}\right|^{2}\right)^{1 / 2}\left(g, k_{\zeta_{n+1}}\right)=\left(\frac{1-\left|\zeta_{n}\right|^{2}}{1-\left|\zeta_{n+1}\right|^{2}}\right)^{1 / 2}\left(1-\left|\zeta_{n+1}\right|^{2}\right)^{1 / 2} g\left(\zeta_{n+1}\right) \\
& <\left\|C_{\phi}\right\| o(1)
\end{aligned}
$$

since $\lim _{|z| \rightarrow 1}(1-|z|)^{1 / 2} f(z)=0$ for $f \in H^{2}$ [3, Theorem 5.9]. So $\|g\|^{2}=$ $\lim \left(g, g_{j}\right)=0$. This contradiction shows $|a|<1$.

Corollary. If $C_{\phi}^{N}$ is compact for some $N>0$, then $\phi(z)$ has a fixed point in $\mathbf{D}$. 
Proof. Because $C_{\phi}^{N}=C_{\phi_{N}}$, for some $\alpha \in D, \phi_{N}(\alpha)=\alpha$, and thus $\phi_{k N}(\alpha)=\alpha$ for positive integers $k$. A periodic sequence converges only if constant, so $\phi(\alpha)=\alpha$.

Theorem 3. If $C_{\phi}^{N}$ is compact for some $N>0$, then $\sigma\left(C_{\phi}\right)=\left\{\phi^{\prime}\left(z_{0}\right)^{n}\right.$ : $n=1,2, \cdots\} \cup\{0,1\}$, where $z_{0}$ is the fixed point of $\phi$.

Proof. By an operator similarity, we may assume the fixed point is at 0 . For if $\tau$ is a linear fractional transformation of $D$ onto $D$ taking $z_{0}$ to 0 , then

$$
C_{\tau} C_{\phi} C_{\tau}^{-1}=C_{\tau \circ \phi \circ \tau^{-1}}
$$

and $\tau \circ \phi \circ \tau^{-1}(0)=0$. By the chain rule, $\left(\tau \circ \phi \circ \tau^{-1}\right)^{\prime}(0)=\phi^{\prime}\left(z_{0}\right)$.

The matrix of $C_{\phi}^{*}$ with respect to the basis $\left\{z^{k}\right\}$ is upper triangular with diagonal entrie s $1, \overline{\phi^{\prime}(0)}, \overline{\phi^{\prime}(0)^{2}}, \overline{\phi^{\prime}(0)^{3}}, \ldots[1]$, so $\phi^{\prime}(0)^{n} \in \sigma\left(C_{\phi}\right)$. Thus $\left\{\phi^{\prime}(0)^{n}: n=1,2, \ldots\right\} \cup\{0,1\} \subseteq \sigma\left(C_{\phi}\right)$.

It remains to show that the only possible eigenvalues for $C_{\phi}$ are 1 and $\phi^{\prime}(0)^{n}$ for some $n$. This is essentially due to Koenig [4]. If $f \circ \phi=\lambda f$, then $f(0)=\lambda f(0)$, so if $\lambda \neq 1, f(0)=0$. Write $f(z)=z^{m} g(z)$, where $g$ is holomorphic in $D$ and $g(0) \neq 0$. It follows that $\phi(z)^{m} g(\phi(z))=\lambda z^{m} g(z)$, and thus $(\phi(z) / z)^{m} g(\phi(z))=\lambda g(z)$. The value of $\phi(z) / z$ at $z=0$ is $\phi^{\prime}(0)$, so $\lambda=\phi^{\prime}(0)^{m}$.

Since the nonzero spectrum of a power-compact operator consists of eigenvalues, the theorem is proved.

\section{REFERENCES}

1. J. G. Caughran, Polynomial approximation and spectral properties of composition operators on $H^{2}$, Indiana Univ. Math. J. 21 (1971/72), 81-84. MR $44 \# 4213$.

2. M. A. Denjoy, Sur l'iteration des fonctions analytiques, C. R. Acad. Sci. Paris 182 (1926), 255-257.

3. P. L. Duren, Theory of $H^{p}$ spaces, Pure and Appl. Math., vol. 38, Academic Press, New York, 1970. MR $42 \# 3552$.

4. M. Koenig, Recherches sur les intégrals de certaines équations fonctionnelles, Ann. Sci. École Norm. Sup. 3 (1884), 1-112.

5. E. A. Nordgren, Composition operators, Canad. J. Math. 20 (1968), 442-449. MR $36 \# 6961$.

6. J. V. Ryff, Subordinate $H^{p}$ functions, Duke Math. J. 33 (1966), 347-354. MR $33 \# 289$.

7. J. H. Shapiro and P. D. Taylor, Compact, nuclear, and Hilbert-Schmidt composition operators on $\mathrm{H}^{2}$, Indiana Univ. Math. J. 23 (1973), 471-496. 
8. J. Wolff, Sur l'iteration des fonctions..., C. R. Ac ad. Sci. Paris 182 (1926), 42-43, 200-201.

DEPARTMENT OF MATHEMATICS, UNIVERSITY OF PUERTO RICO, MAYAGUEZ, PUERTO RICO 00708

DEPARTMENT OF MATHEMATICS, CALIFORNIA STATE UNIVERSITY, LONG BEACH, CALIF ORNIA 90804 (Current address of H. J. Schwartz)

Current address (J. G. Caughran): 809 Dynes Road, Burlington, Ontario L7N 2P7, Canada 